\title{
Prototype of Bus Ticketing Application Using NFC on Android Device
}

\author{
Khan Amjad ${ }^{1}$, Mohammad Mohsin Sheikh², Shaibaz Arkate ${ }^{3}$, Lutful Islam ${ }^{4}$ \\ Student, Computer Engineering, MHSSCOE, Mumbai, India ${ }^{1,2,3}$ \\ Assistant Professor, Computer Engineering, MHSSCOE, Mumbai, India ${ }^{4}$
}

\begin{abstract}
Mobile devices have larger impact in our daily life. The proposed project is concerned with ticketing and identification of the passenger in the public transport service. As seen in various metropolitan cities like Mumbai, Kolkata, Chennai, Bangalore there are various issues and problems related to severe malfunction and security. There are various problems related to public transport like confusion and misunderstanding between the passengers regarding fares, facing the severe problem of traffic jam, having severe security problems in public transport due anti social elements. This proposed project aims to develop a user friendly, interactive automated ticketing system which will deduct the passenger's fare according to the distance travelled as well as detect the passenger's identification. This is only possible by use of Near Field Communication (NFC) cards.
\end{abstract}

Keywords: NFC cards, NFC card reader, Android mobile, Digital bus ticket

\section{INTRODUCTION}

The project is developed using NFC technology. This project provides a NFC system that can identify passengers in public transport as well as it does all
accounting purpose based on travelling fares. Automated and accurate accounting of bus transport can be used to provide useful estimates of the fare of travelling from one bus stop to another as well as the crowd density can be measured inside the bus, but in case of India measuring crowd density is of no use. Near field Communication (NFC) tags is going to be used in this project. Passenger would carry NFC card with them, when they enter into the bus they have to show the NFC card to the Conductor. The conductor will use NFC based android cell phone to read the NFC tag. Based on the distance travelled the cost would be automatically deducted.

Near Field Communication is a wireless technology which allows transmitting data between two devices. NFC commonly integrated within mobile devices. This will allow the device to establish communication with another device sim card. So it can be used as a substitute of wallet in public transportation. Basically NFC has 2 different modes of data transmission:

1. Active NFC Mode: in this mode, source and destination use their self generated radio frequency to communicate.

2. Passive NFC Mode: in passive mode, destination answer request made by source to call modulation scheme. Source does the radio frequency generation.

The project is basically divided into two modules:-

1. Server

- To manage administration

- $\quad$ To manage Conductor/Ticket Checker(TC)

- $\quad$ To manage Passenger

- $\quad$ Renewal of account

2. Android Application

- $\quad$ Conductor Login

- TC Login

- $\quad$ Ticket booking

- $\quad$ NFC Reading

Copyright to IJARCCE

\section{EXISTING SYSTEM}

In existing system there was a pass type ticketing which was fixed for same source and destination and It was of two type monthly and quarterly. The passenger has to visit the bus depot to renew his/her account and NFC tag was used and data was entered to that card and it was scanned in each travel from source to destination and it expires at the end of month or quarter as per account type, but proposed system is quite flexible that is it has to renew as per user's need and source and destination is also flexible, as per the journey cost the amount is deducted from passengers account so it is easy, fast and less cash handling process. So the existing system is not user friendly.

In that either customer can board the bus and then take the ticket or he can use pass for monthly and quarterly for fixed source and destination. Some time change issue also get create. Passenger and conductor both don't have change for ticket amount which creates issue.

This is standard method works in the public transport.

\section{A.}

\begin{tabular}{ccc}
\multicolumn{9}{c}{ III. } & ANDROID OS \\
Now a day's many & mobile devices such as smart
\end{tabular} phones and a tablet computer uses an operating system which is known as android. It is developed by Google. The Android Open Source Project (AOSP) does the maintenance and further development of Android. It has good Linux kernel, middleware layer , includes libraries and application programming interface written in $\mathrm{C}$ and application software running on an application framework which is virtual machine with just-in-time compilation to run compiled java code. There are many Android developers who develop an efficient application that extend the functionality of the existing android 
application. Generally developers write code in a new $B$. version of java. Android apps can be downloaded from different third-party websites or through online stores such as Android Market, the Google app store. The android system uses the Dalivik Virtual Machine to run java based applications. You cannot directly run java class files on android they need to get converted in the Dalivik byte code format.

\section{IV.}

\section{Proposed System}

In proposed system conductor/ticket checker should have .apk file in his/her NFC supported android based device. Conductor/ticket checker can get access to the application by providing his/her username and password. Conductor can perform task like scan NFC card, book ticket, view ticket log. Ticket checker can perform task like scan NFC card and view ticket $\log$.

\section{A. Operations Of The Proposed System}

As soon as the bus arrives at the bus stop, the passenger would board the bus and show the tag to the Conductor. Using android based NFC reader conductor will read the NFC tag. NFC card will have a unique ID number. The card is rechargeable from certain bus depots placed at certain locations of the city. The reader will accept the card if the card has required credit to travel that distance. The reader will read the tag and acquire certain information from the passenger. According to the source $\&$ destination the fare would be deducted from the NFC tag. After the whole day, the individual bus reader will know how much amount has been transferred to the corresponding account and also the information of passengers can be found in the main database. Thus better monitoring and transparency can be provided by cross checking of all those information.

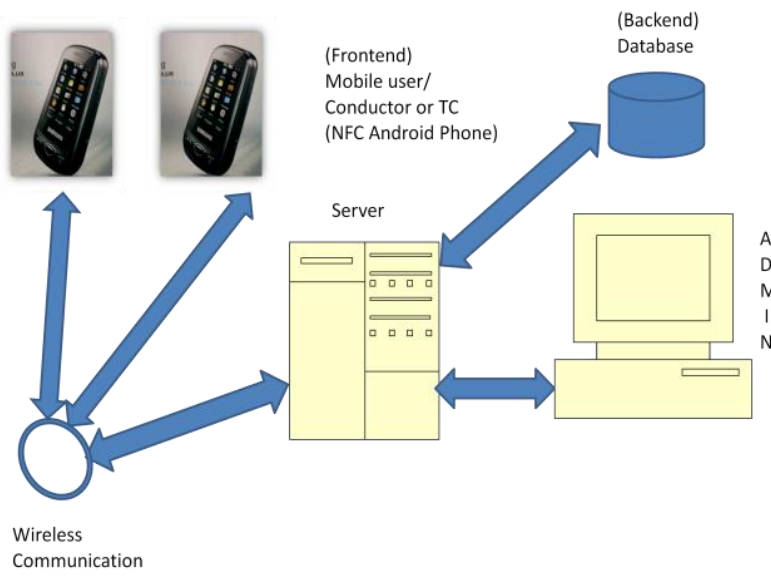

Fig. 1 Architecture Of Proposed System

The architecture of the proposed system consists of the following blocks:

- User frontend android application

- Website/web based application

- Web service application

- Database at the backend to store the records
B. Details Of Hardware And Software

Hardware Requirements:

\author{
$>\quad$ NFC tags \\ NFC based android mobile \\ 2 GB RAM. \\ 160 GB HDD. \\ Intel $1.66 \mathrm{GHz}$ Processor Pentium 4
}

\author{
Software Requirements: \\ Visual Studio 2010 \\ MS SQL Server 2005 \\ SDK for Android 4.2 \\ Eclipse \\ Windows Operating System \\ V. OBJECTIVE
}

It is designed to help in public transport system and passengers to deploy mobile NFC solutions to enhance the efficiency and effectiveness of public transport. It aims to provide better solution to get the potential benefits and to avoid obstacles and considers the deployment of transportrelated mobile NFC services. Mobile NFC ensures to put passengers in control, reducing stress and making travelling by public transport a more pleasurable experience. This user friendly technology, combined with mobile connectivity, makes it easy for passengers to modify their travel plans on the move. Near Field Communication is a radio technology that can transmit data between two devices. Now a days mobile phones are increasingly being integrated with NFC capabilities, enabling new digital services that can greatly increase the passenger experience of public transport. The combination of NFC, an easy-to-use OS and versatile technology, with mobile connectivity could provide many services in the transport sector.

Improve the value and functionality of existing contactless infrastructure

- Better passenger convenience: easy to buy NFC tickets via a mobile connection and not to stand in a queue.

- Cost Effective: Lower sales and distribution costs, combined with possible recycling and environmental benefits

- Feasible: Fast, accurate and transparent ticket validation

- $\quad$ More flexible and interoperable ticket systems: NFC handsets can support multiple ticketing standards, creating a greater experience for passengers

- Interaction: Personalized communication with passengers and promotion of public transport

M-commerce: It provides a good platform for different mobile commerce services like advertising and marketing on android app.

- Efficient and Good Quality: Because of eTicketing it improves the efficiency and effectiveness of the old ticketing process and increases the quality of service. 


\section{A. From The Conductor/Ticket Checker(TC) End:}

- Conductor/TC must have downloaded an .apk file and installing that .apk file in his/ her NFC supported android based device.

- Icon will be created, after clicking on the icon; Bus

Ticketing application will be started.

- Conductor/TC can get the access to system by providing his/her username and password, then conductor can perform tasks like scan NFC card, select source, destination \& create ticket log. Ticket checker scans NFC card and view ticket log. Each NFC card has unique passenger identifier.

\section{B. From The Admin End:}

-Administrator has to launch the website on the server \& also providing link of the .apk file.

- Administrator accesses the website and login the website for these functions/management described below.

- Passenger Management: In this module admin will insert the passengers information on the site also can edit or delete that passenger's entry from the database.

- Conductor/TC Management: In this module admin will insert the conductor/TC's information on the site, can also edit or delete their records from the database.

- Ticket log Management: The admin can delete the ticket logs created by conductor \& can also change the format of the ticket being generated.

- Bus stop Management: In this module admin can manage the different bus stops and add or delete the entry for different bus stops.

\section{SCOPE}

There is always an opportunity for improvement no matter how good the software might be and the same thing goes for our software too. Since we have used asp.net there is a lot of scope for future enhancement

1. Easy Recharge: The user can recharge his account through net banking, pay pal, or from his mobile account without visiting any bus depot.

2. Providing Bus Route and Cost: An interface can also be added in android app that user can see the bus route and cost for a journey from source to destination.

3. Authorization: The photo of the passenger can also be attached to the NFC card so only the authorized person can travel not anyone else.

4. Providing eTicketing approach: All features reflected by the "automation and accuracy" potential of eTicketing for the passenger and ticketing method.

\section{AdVAntages}

- $\quad$ It has user friendly interface.

- Better passenger convenience

- $\quad$ Fast, accurate and transparent ticket validation

- $\quad$ Less cash handling

- $\quad$ Auto Accounting

- Useful data, real time information and pricing

- $\quad$ Flexible source and destination

- $\quad$ No use of papers

Copyright to IJARCCE
- Lower sales and distribution costs, recycling and environmental benefits

- Low cost validation equipment

- $\quad$ More flexible ticket systems

- Enhance the value of existing contactless infrastructure system

\section{CONCLUSION}

Human efforts are reduced because system is completely automated and efficient. The cards being reusable, they are much more convenient and eco-friendly compared to the existing ticketing system. The record of the entire expense of the bus can be provided by the bill printer. Any unwanted things can be avoided as all the person carrying NFC tickets are monitored every time they travel.

\section{ACKNOWLEDGMENT}

We take an opportunity to express an immense gratitude to Mohammed Haji Saboo Siddik College of Engineering, for providing us the opportunity to perform this project. We are also thankful to all anonymous reviewers for their suggestions, good reviews and constructive feedbacks.

\section{REFERENCES}

[1] Near Field Communication: ECMA International Standardizing Information and Communication Systems.

[2] R. Das, "NFC-enabled phones and contactless smart cards 2008 2018," Card Technology Today, vol. 20, Jul./Aug. 2008, pp. 11-13.

[3] NFC in Public Transport -January 2011 http://www.nfc-forum.org Transport Infrastructure\| Fourth International Workshop with Focus on Near Field Communication 978-0-7695-4678-0/12 \$26.00 (C) 2012 IEEE DOI 10.1109/NFC.2012.1413 2012 4th International Workshop on NFC

[4] RFID-based ticketing for public transport system: Perspective megacity Dhaka

[5] http://developer.android.com/index.html

[6] N. F. Inc, - Nfc in public transport. January 2011, last visited on January 19th 2012. [Online]. Available: http://www.nfc-forum.org/ resources/white papers/NFC in Public ransport.pdf

\section{BIOGRAPHIES}

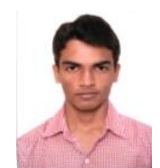

Name: Mr. Khan Amjad

Designation: Student

Department: Computer Engineering

Qualification: B.E.(comp) Pursuing

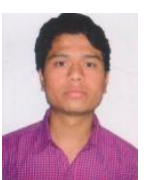

Name: Mr. Mohammad Mohsin Sheikh

Designation: Student

Department: Computer Engineering

Qualification: B.E.(Comp) Pursuing

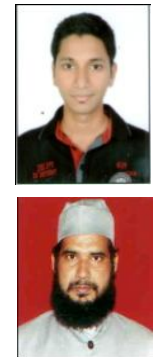

Name: Mr. Shaibaz Arkate

Designation: Student

Department: Computer Engineering

Qualification: B.E.(Comp) Pursuing

Name: Mr. Lutful Islam

Designation: Assistant Professor

Department: Computer Engineering

Qualification: M.TECH(Comp) 